\title{
Structural Relaxation Solutions Formetallic Amorphous Condensed Lattice with Activation Energy Spectrum Model Applied Computational Physics
}

\author{
Kazu.Yamada, Hakodate Natl. \\ College of Technology, Dept. of Production Systems Engineering (Dept. of elec.\&elec.Engng.) \\ 14-1Tokura, HAKODATE, HOKKAIDO, JAPAN, 042-8501 \\ yama@js8.so-net.ne.jp, yama@hakodate-ct.ac.jp
}

\begin{abstract}
Progressformaterial amorphous-devices and specimensfor modification methods are closely correlated with the clearness of elevated thermal constancy and steadfastness of relaxation, additionally general ground characteristic investigations of glass, phase alteration of the liquid-system to glassy amorphous one is an enormous centerpiece in solid state physics on condensed matter. The aims of presentingexaminationare to elucidate numericmathematical answers for construction relaxation procedures concentrating on the activation potential energy in metallic elements based glassy amorphous alloys.Activation drive energy spectrums by calculated with relaxed ratio function were observed and with numerical advances in computer engineering solution methods. It has been concluded that linear ratio function applied computer solutions clear numerical details for relaxationsnumerically of energy peak. It has beenobserved around $160 \mathrm{~kJ} / \mathrm{molthat}$ has good agreement against otherexperimental results.
\end{abstract}

Keywords-component; structural relaxation; amorphous alloy; activation energy spectrum; Differential Scanning Calorimetry; computational physics

\section{INTRODUCTION}

Physical processformaking the overalltypical of glassesaresupposed that the makeover transform of the liquidsystem to glass is a concludingsubject in physical science through onto the span of century. Meanwhile the progress of amorphoussubstantialdiplomacies and specimen alteration methods is thoroughly connected with the conspicuousness of high thermal constancy and constancy of relaxation. The aims of this examination are to elucidate numeric mathematical solutions for construction relaxation processes concentrating on the activation energy amorphous alloys in transition metallic type based. Potentialsactivation for physical relaxation procedure in a metallic type amorphous nearby binary alloysequipped by chilly-extension melt spinning has been explored by Differential Scanning Calorimetry,DSC, with a cyclical method heating performance $[1,2]$ and with numerical advances in Computer engineeringsolution methods[3]. Activate triggering energy for physical relaxation with analtitudinal amount in amorphous substantial wasdeliberated using anentire relaxed proportion role that is contingent on annealing time and temperature. In the current work in amorphous nearby ternaryalloys, the circulatorydistributions for the activation energy spectrum ,AES, by calculated with derivativetype relaxed ratio meaning function were presently observed. An additional consequencewas also recognized that the reverse- phenomena AES classical energy distribution nevertheless the cyclically nano-structural relaxations were in good covenant with the presented experimental results of transition metallic type amorphous alloys. There wasfreshly considerable as to whether the glassy alloys are archetypal of the bulk formed ultra-fine structure [4]. Predominantly $\mathrm{Cu}$ has been shown to be good base element for bulk glassforming alloy with fully glassy sections lately by custom of die injection casting [4]. Alloys for $\mathrm{Cu}$ system have been initiatedto form an amorphous phase over a wide configuration collection. Conversely, adding of $\mathrm{Ti}$ in both these binary systems significantly increased the glass forming ability ,GFA, with the critical diameter for fully amorphous rods being at least approximately an half of $\mathrm{cm}$ for $\mathrm{Cu}-\mathrm{Zr}-\mathrm{Ti}$, and $\mathrm{Cu}-\mathrm{Hf}-\mathrm{Ti}[1,2,4]$. Temporarily the considerate of the physical relaxation procedure is indispensable on the progress of constancy of amorphous alloy systems, as high as in founding steady operational temperature to prevaricate the poverty of asset. Accordingly, concerning in height thermal steadiness of quasistable amorphous resources for $\mathrm{Cu}$ grounded composites, the atomic procedure of diffusion in amorphous composites is still poorly understood as paralleled to that in crystalline composites. Nonetheless, dimensions of diffusivity in amorphous composites have been limited so far for the reason that of the investigational difficulties of determining the tiny diffusivity-constant, habitually less than $10^{-17} \mathrm{~m}^{2} \mathrm{~s}^{-1}$, which are typical of amorphous alloys below their crystallization temperatures [3]. On the current effort, by use of DSC, thermal investigation analysis has been complete to define the active-transprogressions [3], or to appraise whether it signifies thermo-dynamically constant procedure for typical glass-forming amorphous alloys.

\section{CALCULATION PROCEDURE, THEORY WITH} ANNEALING PROCESS USING COMPUTATIONAL DSC [5]

Suppose that in the amorphous substantial therearerelaxation centers that can be designated by detached 2-LevelPotential-wells, in Fig. 1, i.e. an atom or a cluster of atoms couldsubsist in 2-configurations of dissimilar energy, thenevolutions are likely between them via activation. Regardingamorphous substantial quasi-continuous spectra of the restrictions $E$ and $\Delta$ for theTLSscould be predictable. Besides, form and numeral of TLSs are hypothetical to be unchanged. In the currentfigure the relaxing-action reproduces purely apopulation alternateof the TLS in aninspected substantial possessions. 
Opening theyexplore the population alteration of detached TLSs described by $E$ and $\Delta$, which are primarily not in equilibrium. Theydesignate the population of the 1 st state by $n_{1}$, the time derivative is designated by a point, and to be applied1storder kinetics and to be taken into interpretation the possible incomplete changes resulting as fig. 1 theywere gained as,

$$
n_{1}^{\prime}=v_{E+\Delta} n_{2}-v_{E} n_{1}
$$

Where $v_{E}$ is the degree of the change through a potential barrier of energy $E$. An Arrhenius formulation is revealed to $v_{E}$,

$$
v_{E}=v_{0} \exp \left(-\frac{E}{\mathrm{k} T}\right) \quad .
$$

Answeringthe exceeding the time derivative differential equation,

$$
\begin{gathered}
n_{1}(t)=\left[n_{10}(E, \Delta)-n_{1 \infty}(\Delta, T)\right] \theta_{v_{0}}(E, T, t) \\
+n_{1 \infty}(\Delta, T)
\end{gathered}
$$

Where they familiarize, for suitability, anevent exemplifying the annealing procedure and instantaneously droplet its feeble $\Delta$ requirement as underneath,

$$
\begin{gathered}
\theta_{v_{0}}(E, T, t) \equiv \exp \left[-v_{0} t \exp \left(-\frac{E}{\mathrm{k} T}\right)\right] \\
n_{1 \infty}(\Delta, T)=\frac{1}{1+\exp \left(\frac{\Delta}{\mathrm{k} T}\right)} .
\end{gathered}
$$

An as-quenchedcondition,

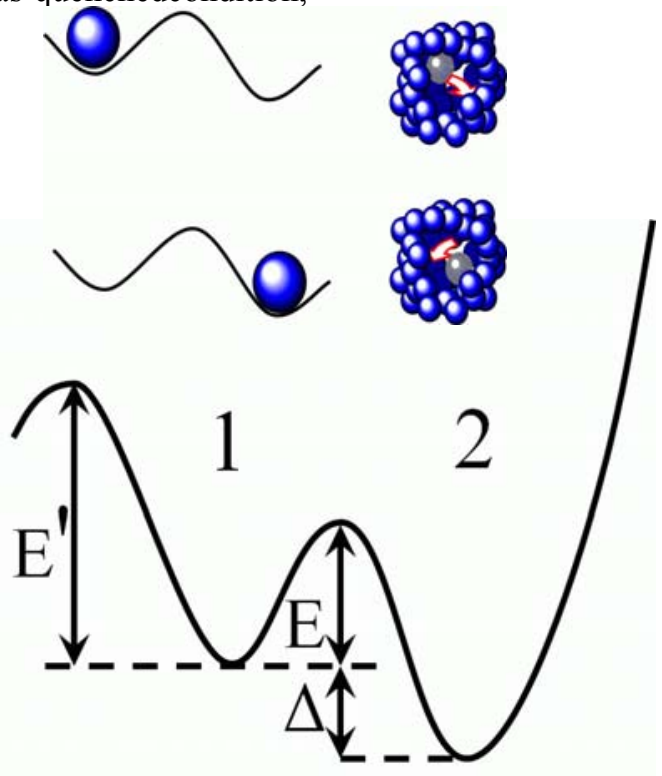

Figure 1. Illustration of relaxing center and energy-level for corresponding a 2-Level system (TLS).
$n_{10}(E, \Delta)=n_{1 \infty}\left(\Delta, T_{g}\right)$ could be inscribed disregarding the subdued energy TLSroom temperature equilibrated during storage. While $n_{2}(T)=1-n_{1}(T)$, they considered $n_{1}$ in the break of this behaviorand also return to aninitial.

With the intention of realize what limitations will be forced on an ns in order that it could be deliberated as aunopened system, it would be permitted for the excite of particles with space outside of a TLS ( $E^{\prime}$ is limited finite on fig. 1). They could be revealed simply that if $E^{\prime}-E \geqslant$ $5 \mathrm{k} T$ therelation fora time const.of an internal TLS changeoverto that of excitesframe out of the TLS is $\preccurlyeq$ $10^{-2}$, accordingly the TLSscould be deliberated asunopened system. For instance $T=300^{\circ} \mathrm{C}, E^{\prime}-E \cong 0.2 \mathrm{eV}$ is necessary for TLSs to beun-opened system. ExperimentationTLSs as E assessments among $1 \gtrless 2 \mathrm{eV}$ are mostly excitation. Fluctuatedoscillations of $0.2 \mathrm{eV}$ in order of admiration to $E$ could be predictable in a dis- ordered system, A Böhönyeyet al have been continuously explainedas above.

So as to associate a smart type for quantity with the experiment they ought to trace asubjective anneal-practice. They estimated asubjective annealprocedure $T(t)$ with a sequence of slight dynamics (fig. 2). It should be written the role of single TLS, regarded asparams. $E, \Delta$, to a reduction. The structuralpossessions alteration amongst the $i$ th and $j$ thon anneal-stages, named elementary-relaxation, $R_{\text {elementary }}^{i j}$ is assumed by

$$
R_{\text {elementary }}^{i j}=\Delta P=\left(n^{(i)}-n^{(j)}\right) C
$$

When $n^{(i)}$ is a residents subsequently the $i$ th anneal-stage and $C$ is a combination issue linking the residents alteration to the substantial assets modification. Afterward the primary anneal-stage (temperature $T_{1}$ for time $t_{1}$ ) the residents could be inscribed allowing to (3),

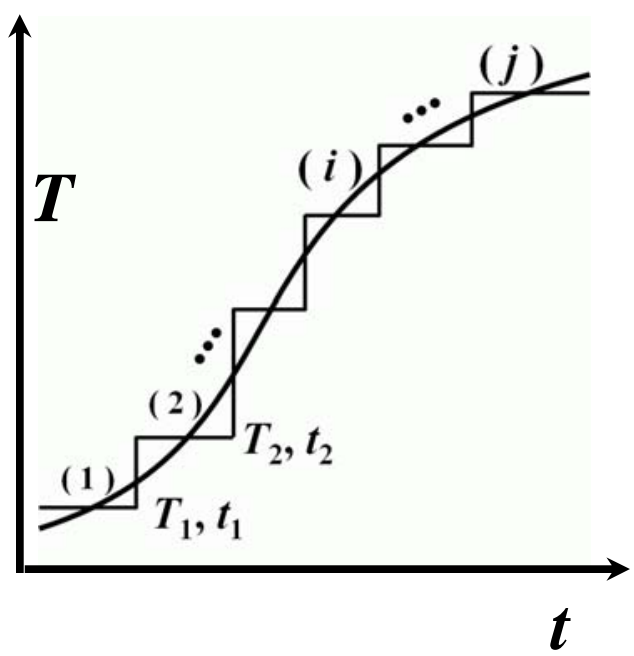

Figure 2. Schematic timevs temperature illustration of an arbitrary annealing procedure approximated by a series of slight kinetics. 


$$
\begin{gathered}
n^{(1)}=\left(n_{0}^{(1)}-n_{\infty}^{(1)}\right) \theta^{(1)}+\theta_{\infty}^{(1)}(7) \\
n^{(k)}=\left(n_{0}^{(k-1)}-n_{\infty}^{(k)}\right) \theta^{(k)}+\theta_{\infty}^{(k)} .
\end{gathered}
$$

Circumstance of TLSs spectra, the fundamental TSL influences would be condensationwhich is achievabletrails from the hypothesis of no relations amongdetached TLSs. Accordingly,

$$
\begin{gathered}
R^{i j}=\int_{0}^{\infty} \int_{0}^{\infty} q(E, \Delta) \overline{C(E, \Delta)}\left[n^{(i)}(E, \Delta, T)\right. \\
\left.-n^{(j)}(E, \Delta, T)\right] \mathrm{d} E \mathrm{~d} \Delta
\end{gathered}
$$

$q(E, \Delta) \mathrm{d} E \mathrm{~d} \Delta$ is aquantity of the TLS having among limitations $E$ and $E+\mathrm{d} E$ and $\Delta$ and $\Delta+\mathrm{d} \Delta$. Acombination period, $\overline{C(E, \Delta)}$, is amiddling worth. TLS would beconsidered by the similar constraints $E$ and $\Delta$ will be able to characterize altered atomic procedures providing different influences to astructuralpossession change. Supposing

$$
q(E, \Delta)=q(E) \quad \text { if } \Delta<5 \mathrm{k} T
$$

even $T$ is the supreme temperature in an experimentation, consequence TLSs forseveral $\Delta$ assessment fewer than $5 \mathrm{k} T$ could be originate ofidentical possibility in thecondensed matter. Current hypothesis is observable since the TLSs with $\Delta>5 \mathrm{k} T$ don't subsidize to the changeable consequence [5], and for the reason that the closely equivalent allocation of $q(E, \Delta)$ of $\Delta$ is mentioned the amorphous construction.

Regarding $\Delta$ requirement of acombination issue, they survey anopportunity of 2expectations [5].

An extra assumption oftwo of them, presuming $\overline{C(E, \Delta)}=\overline{C(E)} \Delta$ they come to be

$$
\int_{0}^{\infty} n_{\infty}^{(i)}(\Delta, T) \Delta \mathrm{d} \Delta \propto T_{i}^{2}
$$

The calculation to achievement $n^{(i)}(E)$ is now straightforward. Note that in DSC measurements $\overline{C(E, \Delta)} \propto \Delta$ [5], sighted as the participation for a TLS for a limitation $\Delta$ to the annealingprogress is proportionate to $\Delta$. (In the case of other material magnitudes it is problematic to provide the $\overline{C(E, \Delta)}$ occupation.)

The relaxation between the ithand jthstage can be transliterated in therule

$$
R^{i j}=\int_{0}^{\infty} p(E) \theta^{i j}(E) \mathrm{d} E .
$$

Where $\quad p(E)=\overline{C(E)} q(E) \quad$ is distinguishing for the substantial $\theta^{i j} \equiv n^{(i)}(E)-n^{(j)}(E)$ is distinguishing for the anneal. $\theta^{i j}(E)$ could be estimated the anneal procedure $T(t)$ usingan experimentation. Nevertheless, but a comparatively slight dynamism intermission is removed out by selecting an appropriate $\theta^{i j}(E)$, that is habitually situation at what time determining dynamic, $p(E)$ could be deliberated to be steady in the selected intermission. The postulation is no less than a good preliminary socket and theyconcern them in anassessment procedure of their statistics. They are probable to plot $p(E)$ onthehuge dynamism intermission by dynamic dimensions upondissimilar heats.

They are worth revealing when (12) is wide-ranging authority in the outline of the assumed archetypes. Not merely for $p(E) \cong$ const, although for $p(E) \cong \delta\left(E_{0}\right)$ (distinct spectrum). The exact form of $\theta^{i j}(E)$ requireaccount in every case, A Böhönyey have been explained over-refine in their instrument [5].

The residents of an congregation of presentedarchetypes, activation energy spectra, $\mathrm{AE} \mathrm{S}$, in physical relax-action procedures, J. A. Leake[6,7] et al define the singularities of structural accessible and flexible possessions with suitable agreement among theory. The concept suppose exponentialelement closely equivalent one-dimension for chemical reaction kinetics of J-Avramiequality.Hence, a hypothetical ideal for the relax procedure in amorphous resources onto the foundation of spectra of obtainable procedures with anallocation of activation energy was suggested. In archetypes, overall alteration in the deliberate possessions, $\Delta P$ is agreed with

$$
\Delta P=\int_{0}^{E} p(E) \mathrm{d} E=.
$$

Collection of activevitalityenergy $E$ to $E+\mathrm{d} E$ throughout the physical relax-procedure, the entire accessible property $p_{0}(E)$ deviations are

$p(E) \mathrm{d} E=p_{0}(E)\left[1-\exp \left\{-v_{0} t \exp \left(-\frac{E}{\mathrm{k} T}\right)\right\}\right] \mathrm{d} E$.

Where $v_{0}$, an Debye-frequency order $\left(v_{0} \cong 10^{12} \mathrm{~Hz}\right)$. Primak [8] modifiedEq. (14) as

$$
p(E)=p_{0}(E) \theta(E, T, t) .
$$

Where $\theta(E, T, t)$ arewell-defined as the characteristic anneal-utility. Accordingly, the formulas of $\theta(E, T, t)$ areration of the percentage of obtainable procedures edge of the dynamism $E$. Percentage as $\theta(E, T, t)$ has donated to the relax-possessions on annealing temperature $T$ after time $t$. In an alternative document [9], a procedure furthermost simplify supposition, the formulas $\theta(E, T, t)$ could be interchanged asa step formula at an energy $E_{0}(T, t)$,

$$
E_{0}=\mathrm{k} T \ln \left(v_{0} t\right) \quad .
$$

At that time $E_{0}$ vicissitudes 0 to 1 above one-step temporarily $\theta(E)$ changes over a slight assortment of $E$ and $T$. But in the abridged intentions, the $E_{0}$ will be practical. In opposition,contemporary effort for estimate consuming definite first derivative relax-ratio, like this

$$
\frac{d \theta(E, T, t)}{d E}=
$$




$$
\left\{v_{0} t \exp \left(-\frac{E}{\mathrm{k} T}\right)\right\}\left(-\frac{E}{\mathrm{k} T}\right) \exp \left\{-v_{0} t \exp \left(-\frac{E}{\mathrm{k} T}\right)\right\}
$$

Moreover the standardisation for the rectilinear meaning was put on. It is entitled standardised first derivate-relaxratio in organized current presenting exertion.

\section{RESULTS AND DISCUSSIONS}

Some amorphous-substantialconstructions and sample modificationsarestrictly connectedclearness of extraordinary thermal immovability orsteadiness of relax. Wide-ranging characteristic into glass, alterationsof the liquid-system to glassare gigantic condensed matter coming over the physical science. To elucidate arithmetical explanations for construction relaxation procedures concentrating over the activevitality energy in metal grounded amorphous components. Activation energy varieties by analyzed with relaxed ratio function were detected and with arithmetical advances in computer engineering solution methods shown in Figure 3. It has been concluded that linear ratio function $f(T, t, E, G P)$ applied computer resolutions clear numerical details for relaxations arithmetically of energy peak. Where GP means DSC of endo/exo-thermic heat for annealing process approximated by a series of small summations value between without relaxed and with relaxed state upon a specimen(without relaxed specimen minus with relaxed one). It has been observed activation energy spectrum having peak around $160 \mathrm{~kJ} / \mathrm{mol}$ that has good agreement against other typicalexperimental results on metallic amorphous alloys.

\section{ACKNOWLEDGMENT}

Author would like to show the appreciation toDrI A Figueroa (Universidad NacionalAutonoma de Mexico) for collaboration tocurrentwork.

\section{REFERENCES}

[1] Kazu. Yamada,, N. Shinagawa, M. Sogame, I.A. Figueroa, H.A. Davies, "Structural Relaxation Process in Cu-Hf-Ti," Defect and Diffusion Forum, vol. 283-286, pp. 533-538, March 2009.

[2] Kazu. Yamada,, N. Miura, A. Yamamoto, I.A. Figueroa, H.A. Davies, "Structural Relaxation Process by Addition of B in Cu-Hf-Ti," Defect and Diffusion Forum, vol. 297-301, pp. 702-707, March 2010.

[3] Kazu.Yamada, "Numerical Solutions for Structural Relaxation of Amorphous Alloys," Chapter 15, pp. 343-364 (InTech book),January 2013.
[4] I. A. Figueroa, H. A. Davies, I. Todd and K. Yamada, "Formation and Thermal Stability of Cu-Hf-Ti-M Glassy Alloys," Advanced Engineering Materials, vol. 9, pp.496-499, June 2007.

[5] A Böhönyey and L F Kiss, "A quantitative study on reversible structural relaxation of metallic glasses," J. Phys. Condens. Matter,vol. 3, pp. 4523-4531, 1991.

[6] J. A. Leake, E. Woldt, and J. E. Evetts, "Gaussian Activation Energy Spectra in Reversible and Irreversible Structural Relaxation,"Mater. Sci. Eng., vol. 97, pp. 469-472, 1988.

[7] M. R. J. Gibbs, J. E. Evetts and J. A. Leake, "Activation energy spectra and relaxation in amorphous materials," J. Mater. Sci., vol. 18, pp. 278-288, 1983

[8] W. Primak, "Kinetics of processes Distributed in Activation Energy," Phys. Rev., vol. 100, pp. 1677-1689, 1955.

[9] Y. Takahara, A. Morita, T. Takeda and H. Matsuda,"Evaluation of Activation Energy Spectra for Structural Relaxation in Amorphous Fe-Ni-B-Si Alloys by AES Model,” J. Japan Inst. Metals, vol. 54, pp. $752-757,1990$

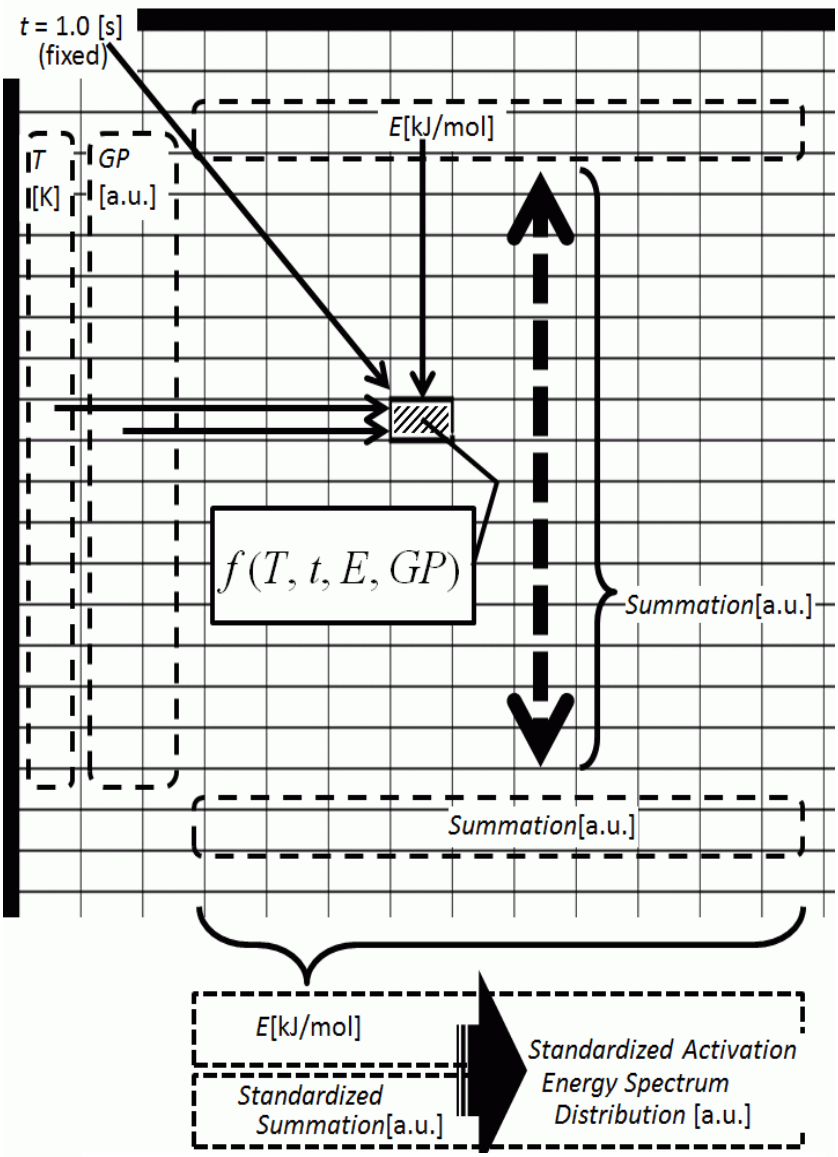

Figure 3. Schematic calculations process on a worksheet diagram of arbitrary samples as $G P$ of endo/exothermic heat for annealing process approximated by a series of small summations. 